\title{
Filtraje Robusto DE SEÑAles DÉBILES DE FENÓMENOS REALES
}

\section{ROBUST FILTERING OF WEAK SIGNALS FROM REAL PHENOMENA}

\author{
Valeri Kontorovich ${ }^{1, *}$, Fernando Ramos-Alarcón ${ }^{1}$
}

\section{Resumen}

En un gran número de escenarios de la vida real se requiere procesar señales de interés que se encuentran muy inmersas en medio de ruido de fondo: señales tectónicas de las entrañas de la Tierra, otras provenientes del lejano cosmos, de telemetría biomédica, acústicas lejanas, interfaces neuronales no invasivas, etc. El propósito de este trabajo es presentar la descripción de una plataforma robusta y eficiente para hacer filtraje en tiempo real de señales muy inmersas en ruido (bastante débiles) y de naturaleza muy diferente. La estrategia propuesta se basa en dos principios: el modelado de las señales de los fenómenos físicos mediante procesos caóticos y la aplicación de estrategias de filtraje basadas en la teoría de sistemas dinámicos no lineales. Tomando como caso de estudio señales sísmicas, señales de electrocardiogramas fetales, señales de tipo voz y señales de interferencias de radiofrecuencia, este trabajo experimental muestra que la metodología es eficiente (error cuadrático medio menor al $1 \%$ ) y robusta (la estructura de filtraje, basada en filtro de Kalman, es invariante ante diferentes señales fenomenológicas). La metodología presentada resulta ser muy atractiva para aplicaciones prácticas para la detección de señales débiles en tiempo real por su alta precisión de filtraje con una mínima complejidad computacional y tiempos de procesamiento muy cortos.

Palabras clave: caos, filtraje no lineal, sistemas dinámicos, filtro de Kalman, señales débiles, señales reales.

\section{Abstract}

In a large number of real-life scenarios it is required to process desired signals that are significantly immersed into background noise: tectonic signals from the entrails of the earth, signals coming from the far away cosmos, biometric telemetry signals, distant acoustic signals, noninvasive neural interfaces and so on. The purpose of this paper is to present the description of a robust and efficient platform for the real time filtering of signals deeply immersed in noise (rather weak signals) with rather different nature. The proposed strategy is based on two principles: the chaotic modelling of the signals describing the physical phenomena and the application of filtering strategies based on the theory of non-linear dynamical systems. Considering as a study case seismic signals, fetal electrocardiogram signals, voice-like signals and radio frequency interference signals, this experimental work shows that the proposed methodology is efficient (with mean squared error values less than 1\%) and robust (the filtering structure remains the same although the phenomenological signals are drastically different). It turns out that the presented methodology is very attractive for the real time detection of weak signals in practical applications because it offers a high filtering precision with a minimum computational complexity and short processing times.

Keywords: Chaos, Non-Linear Filtering, Dynamic Systems, Kalman Filter, Weak signals, Real Signals.

\footnotetext{
$\overline{1, *}$ Departamento de Ingeniería Eléctrica, Sección de Comunicaciones, Centro de Investigación y Estudios Avanzados del Instituto Politécnico Nacional (CINVESTAV-IPN), México. Autor para correspondencia valeri@cinvestav.mx (D) http://orcid.org/0000-0002-1307-3001, (D) http://orcid.org/0000-0002-8235-4886
}

Recibido: 11-09-2019, aprobado tras revisión: 11-12-2019

Forma sugerida de citación: Kontorovich, V. y Ramos-Alarcón, F. (2020). «Filtraje robusto de señales débiles de fenómenos reales». InGENIUS. N. ${ }^{\circ}$ 23, (enero-junio). pp. 109-119. DOI: https://doi.org/10.17163/ings.n23.2020.10. 


\section{Introducción}

El procesamiento de señales es básico para muchas áreas de ciencia e ingeniería. Una de las etapas fundamentales para cualquier algoritmo de procesamiento de señales es el filtraje, es decir, eliminar (filtrar) el ruido de fondo que acompaña a la señal bajo estudio antes del correspondiente procesamiento de señales.

Para contrarrestar los efectos del ruido existen diversas estrategias de filtraje de señales. Las metodologías convencionales de filtraje (basadas en procesamiento estadístico) resultan bastante efectivas cuando la relación señal a ruido (SNR) es mayor o igual a uno, SNR $\geq 1$, o bien SNR $\geq 0 \mathrm{~dB}$ si se expresa en decibeles (es decir, magnitud del ruido menor o igual a la magnitud de la señal de interés).

En muchas aplicaciones prácticas se procesan señales con ruido cuando la magnitud de este último es mayor que la magnitud de la señal de interés, SNR $<1$. Para el filtraje de señales en estas situaciones las estrategias convencionales han sido adaptadas o bien se han creado metodologías novedosas (basadas en procesamientos iterativos, wavelets, etc.). El costo de lograr el filtraje de señales en estas condiciones es que el procesamiento en tiempo real se ve ligeramente afectado.

El proceso de filtraje es un gran desafío cuando se requiere la detección de señales muy débiles (magnitud del ruido mucho mayor que la magnitud de la señal deseada, es decir, SNR « 1 o bien SNR « $0 \mathrm{~dB}$ ), por ejemplo, señales de sistemas solares lejanos, actividad cardíaca fetal, pequeños sismos precursores de terremotos, señales de voz inmersas en ruido de fondo, señales de información de radiofrecuencia, interfaces neuronales no invasivas, etc. El tema de detección de señales débiles no es realmente nuevo y es posible encontrar una gran cantidad de publicaciones (ver [1-6] solo por citar algunas referencias) que abordan este tema con diversas metodologías y para diferentes fenómenos. Se emplean técnicas que van desde diferentes esquemas de filtraje (adaptivo, tiempo-frecuencia, FIR, IIR, etc.), lógica difusa (fuzzy logic), sistemas caóticos, resonancia estocástica, hasta diferentes estrategias de descomposición (modo empírico, wavelets, señales ortogonales, etc.). Lo novedoso para el caso del presente trabajo es la aplicación de señales caóticas como modelos de los fenómenos reales basándose en la teoría de sistemas dinámicos determinísticos, no lineales.

Con una historia de más de 50 años, la teoría de sistemas dinámicos [7-9] es uno de los pilares de muchas áreas científicas, como física, control automático, comunicaciones, etc.; particularmente es muy relevante para el filtraje mediante el célebre filtro de Kalman (propuesto por Rudolph E. Kalman en 1960), el cual permite un filtraje muy preciso considerando que la señal deseada es un sistema dinámico lineal bajo la influencia de ruido blanco aditivo gaussiano (AWGN) y desde su invención hasta la fecha cuenta con un sinnúmero de aplicaciones prácticas recientes y añejas (por ejemplo, $[10,11]$ ).

El filtro extendido de Kalman (EKF) permite aplicar la misma metodología para el caso de sistemas dinámicos no lineales, gracias a que en cada ciclo de operación se lleva a cabo un proceso de linealización (cálculo jacobiano de las correspondientes ecuaciones no lineales) tomando como referencia el punto corriente (muestra actual) de operación.

¿Cómo lograr, a partir de la teoría de sistemas dinámicos no lineales, una estrategia efectiva de filtraje para señales débiles de fenómenos físicos diametralmente diferentes? Una de las opciones que se explora en este trabajo es el modelaje de las señales fenomenológicas como señales generadas por atractores caóticos, i. e. por sistemas dinámicos no lineales, pero deterministas. En este caso la aplicación del concepto de sistemas dinámicos permite dos cosas muy útiles: modelar las señales reales como procesos formalmente determinísticos y aplicar todos los resultados de filtraje basados en la teoría de sistemas dinámicos.

Usar modelos caóticos para señales reales resulta original y bastante eficaz, como se muestra más adelante, a pesar de que como tal el modelaje de fenómenos reales por medio de señales caóticas se ha usado durante más de 50 años en áreas tales como sismología $[1,2,12]$, teoría estadística de las comunicaciones $[13,14]$, telemetría biomédica $[15,16]$, procesamiento de señales submarinas [3], y también en muchas áreas relacionadas a física aplicada [17].

En este trabajo se presenta un filtraje efectivo (cuyos aspectos teóricos se desarrollaron en $[18,19]$ ) en el sentido de una alta precisión en términos de valores muy pequeños del error cuadrático medio normalizado (NMSE $<1 \%$ ). La normalización del error cuadrático medio (MSE) se considera en relación con la varianza de la señal fenomenológica. Por otro lado, el filtraje es también robusto en el sentido de que, para señales de entrada provenientes de diferentes fenómenos físicos, tanto la estructura del filtro como su precisión (valores del NMSE) son prácticamente invariantes.

El modelaje caótico es muy útil porque casi todos los algoritmos de filtraje cuasióptimo se caracterizan por tener una alta precisión (valor muy bajo del NMSE) y una complejidad computacional muy baja. Los detalles teóricos y las demostraciones de estas propiedades se desarrollaron en $[18,19]$ donde el lector interesado puede revisarlos. En este trabajo se aplican y se extienden al terreno práctico las ideas del filtraje no lineal (publicadas en [19]), presentando solo los detalles experimentales de escenarios físicos con diferentes fenomenologías, en concreto señales sísmicas, señales cardiacas (ECG), señales del tracto vocal y señales interferentes de radiofrecuencia (RFI). 


\section{Materiales y métodos}

El desarrollo de este trabajo se centra en dos elementos básicos. Por un lado, métodos desarrollados previamente que dan el sustento teórico riguroso y, por otro lado, programación de código en MatLab para crear un banco de pruebas. El código MatLab se desarrolla a partir de las ecuaciones de filtraje descritas más adelante. En el aspecto teórico, se emplean señales caóticas tanto para el filtraje de sistemas dinámicos no lineales como para modelar señales fenomenológicas. Dado que la idea es obtener algoritmos que puedan ser implementados en una plataforma de cómputo, es importante establecer la complejidad del filtraje dinámico no lineal en términos de operaciones o cálculos computacionales.

\subsection{Filtraje y modelaje caótico}

Un proceso caótico se define a partir un conjunto de ecuaciones diferenciales ordinarias y sus correspondientes parámetros, es decir, es un proceso determinista [17]. En el espacio de fase un proceso caótico conforma una trayectoria orbital con la peculiar característica de que ninguna de todas las posibles trayectorias pasa dos veces por exactamente el mismo lugar [17]. Adicionalmente, un proceso caótico es sensible a cambios en las condiciones iniciales, es decir, dos realizaciones de un mismo proceso caótico cuyas condiciones iniciales difieren en un valor arbitrariamente pequeño son completamente decorrelacionadas en el mediano y largo plazo [17].

A pesar de ser determinista, un proceso caótico genera realizaciones de procesos, que se describen como estocásticos y es justamente la naturaleza deterministaestocástica la que explotamos para generar estrategias de filtraje (con ecuaciones deterministas) y modelar procesos físicos (con realizaciones estocásticas).

Un proceso caótico vectorial $\mathrm{x}(\mathrm{t})$ puede ser generado mediante la siguiente ecuación diferencial ordinaria, ODE:

$$
\dot{\mathbf{x}}=\mathbf{F}(\mathbf{x}, t)
$$

con condición inicial $\mathbf{x}(t 0)=\mathbf{x} 0 ; \mathbf{F}(\cdot)$ es una función vectorial variante en tiempo (representa las ecuaciones caóticas). En nuestro caso consideraremos $\mathbf{F}(\mathbf{x}, t)$ tomando como ejemplo las ecuaciones de los atractores caóticos de Rossler, Lorenz y Chua:

Rossler

$$
\begin{array}{r}
x_{k+1}=x_{k}+T_{s}\left(-y_{k}-z_{k}\right) \\
y_{k+1}=y_{k}+T_{s}\left(x_{k}-0.2 y_{k}\right) \\
z_{k+1}=z_{k}+T_{s}\left(0.2-z_{k}\left(5.7-x_{k}\right)\right)
\end{array}
$$

Lorenz

$$
\begin{array}{r}
x_{k+1}=x_{k}+T_{s}\left(10\left(x_{k}-y_{k}\right)\right) \\
y_{k+1}=y_{k}+T_{s}\left(28 x_{k}-y_{k}+x_{k} \cdot z_{k}\right) \\
z_{k+1}=z_{k}+T_{s}\left(-\frac{8}{3} z_{k}+x_{k} \cdot y_{k}\right)
\end{array}
$$

Chua

$$
\begin{array}{r}
x_{k+1}=x_{k+1}+T_{s}\left[9.205\left(y_{k}-U\left(x_{k}\right)\right)\right] \\
y_{k+1}=y_{k+1}+T_{s}\left[x_{k}-y_{k}+z_{3}\right] \\
z_{k+1}=z_{k+1}+T_{s}\left[-14.3 y_{k}\right]
\end{array}
$$

donde $U\left(x_{k}\right)=m_{1} x_{k}+\frac{1}{2}\left(m_{0}-m_{1}\right)\left[\left|x_{k}+1\right|-\left|x_{1}-1\right|\right.$, $m_{0}=-\frac{1}{7}, m_{1}=\frac{2}{7}$ y $T_{S}$ es el tiempo de muestreo. El algoritmo de filtraje que se usa (descrito un poco más adelante) es el filtro de Kalman discreto, por eso (2)-(4) se introducen en forma discreta.

En el bloque de filtraje se desconoce las condiciones iniciales de la señal bajo procesamiento, lo cual produce efectos de incertidumbre (divergencia) que pueden ser mitigados al incluir un «ruido de proceso» aditivo en la Ecuación (1). Así, se transforma la ODE en una ecuación diferencial estocástica, SDE que da origen a un proceso n-dimensional estocástico Markoviano:

$$
\dot{\mathbf{x}}=\mathbf{f}(\mathbf{x}(t))+\varepsilon \xi(t)
$$

donde $\mathbf{f}(\mathbf{x}(t))$ es análoga a $\mathbf{F}(\mathbf{x}, t)$ en (1). La influencia de una fuente externa débil de ruido blanco se denota mediante $\xi(t)$; las intensidades del ruido se dan en forma de matriz $\varepsilon=\left[\varepsilon_{i j}\right]^{n x n}$.

Al usar la SDE (5) como un modelo para el caos, la primera estrategia que viene inmediatamente a la mente es el filtraje no lineal de señales caóticas desarrollado rigurosamente en $[18,19]$ y que se basa en las ecuaciones de Stratonovich-Kushner (SKE) [7,8], con las cuales se puede describir la ecuación dinámica de la función de densidad de probabilidad (PDF) a posteriori del caos $x(t)$. Nótese que la evolución temporal de la PDF a posteriori para $x(t)$ está completamente caracterizada por la SKE, sin embargo, no tiene solución analítica exacta. Una de las pocas excepciones es usar una SDE lineal, es decir, el bien conocido algoritmo del filtro de Kalman. Justamente por esta razón es que los algoritmos de filtraje no lineal prácticamente siempre se simplifican haciéndolos cuasióptimos o incluso cuasilineales.

Uno se podría preguntar: ¿qué ventajas ofrece el modelaje caótico para el filtraje de señales débiles? Resulta ser que la solución de las SKE para la ODE dinámica del caos (1) presenta propiedades de singularidad cuando la solución está prácticamente sintonizada con el caos determinístico en (1) independientemente del valor de la SNR [18]. 
Un conjunto importante de algoritmos de filtraje cuasi lineal aplica la aproximación gaussiana local (LGA) para la PDF a posteriori $[8,19]$ lo cual resulta ser idóneo para aplicaciones en tiempo real. Algunos de dichos algoritmos son:

- Filtro extendido de Kalman (EKF)

- Filtro de Kalman desnaturalizado (UKF)

- Filtro de Kalman en quadratura (QKF)

- Filtro en cuadratura Gauss-Hermite (GHF)

- Filtro condicionalmente óptimo, etc.

Nótese que la diferencia entre los algoritmos basados en la LGA depende solamente de cómo se escoge la ubicación del estimado instantáneo de $x(t)$. Para el caso de una alta precisión de filtraje todos los algoritmos que aplican LGA [18] pueden ser exitosamente aproximados mediante el EKF porque el valor correcto del proceso filtrado y el punto de referencia para la aplicación de la aproximación gaussiana son obviamente muy cercanos.

Todos estos algoritmos de filtraje tienen, dada una cierta SNR, diferente precisión y una complejidad computacional completamente diferente para una calidad de filtraje preestablecida. Al seleccionar algún algoritmo de filtraje específico para un escenario concreto, es necesario considerar, como posibles criterios, el NMSE junto con la complejidad computacional.

\subsection{Complejidad computacional}

En aplicaciones del mundo real la complejidad computacional de los algoritmos cuasilineales es fundamental. Para el caso concreto de los algoritmos EKF, UKF, QKF y GHF la complejidad computacional se puede analizar en términos de operaciones de sumas y multiplicaciones, descomposición Cholesky, propagación no lineal y cálculo jacobiano. Nuestra evaluación en estos términos se muestra en la Tabla 1.

Tabla 1. Complejidad computacional

\begin{tabular}{ccccc}
\hline & EKF & UKF & GHF & QKF \\
\hline $\begin{array}{c}\text { Sumas } \\
\text { Multiplicaciones }\end{array}$ & 15 & 50 & 25 & 25 \\
$\begin{array}{c}\text { Descomposición } \\
\text { Cholesky }\end{array}$ & 1 & 2 & 2 & 2 \\
$\begin{array}{c}\text { Propagación } \\
\text { no lineal }\end{array}$ & 0 & 15 & 21 & 6 \\
Cálculo jacobiano & 1 & 0 & 0 & 0 \\
\hline
\end{tabular}

Se puede notar que el UKF presenta una mayor complejidad, mientras que el EKF es el menos complejo. El EKF podría degradarse debido al cálculo jacobiano (evaluación de derivadas parciales) si las ecuaciones del atractor son sofisticadas. Sin embargo, para los modelos de las fórmulas (2)-(4), la estructura de filtraje basada en el EKF es la mejor opción. Como se mostrará más adelante, para detección de señales débiles reales se puede lograr una fidelidad bastante aceptable en todos los casos prácticos usando una estructura de filtraje basada en el EKF que internamente usa modelos caóticos tipo (2)-(4).

Como alternativa a los algoritmos cuasilineales del EKF, donde la linealización se actualiza instantáneamente, se puede buscar una solución robusta y de baja complejidad computacional usando una «linealización fija» (con una matriz de linealización predefinida para el problema específico bajo estudio) en vez de la linealización instantánea. De hecho, eso significa que se estaría usando el filtro estándar de Kalman (SKF) [7-11] en vez del EKF y por consecuencia, aunque se tendría una menor complejidad, se tendrían también pérdidas en la precisión del filtraje. Sin embargo, hay que tener en cuenta que la LGA de la PDF a posteriori supone que de hecho todas las componentes del modelo son casi lineales y por lo tanto las pérdidas de precisión podrían ser moderadas.

Nótese que cuando los datos de entrada son variantes es muy común que los algoritmos de filtraje cuasióptimo apliquen estrategias de linealización.

\subsection{Procesamiento multimomento}

Para mejorar fidelidad del filtraje se requiere aprovechar toda la información disponible en la señal bajo procesamiento. Para esto en la metodología de filtraje, aplicando las ecuaciones de SKE, tiene sentido incorporar información adicional (en diferentes instantes de tiempo secuenciales) de la señal compuesta que se ha recibido; es decir, se debe considerar información en forma de bloques (en diferentes instantes de tiempo, es decir, procesamiento multimomento). Los algoritmos multimomento se implementan a través de la generalización de las SKE usando datos mutimomento.

Los algoritmos de filtraje multimomento resultan poco prácticos para implementaciones en tiempo real ya que el retardo debido al procesamiento de muestras de diferentes instantes de tiempo es significativo. Para lograr un compromiso entre complejidad y aumento de precisión de filtraje es razonable considerar el procesamiento de solo dos muestras adyacentes. Este procesamiento se conoce como régimen de dos momentos (2MM), es un caso especial del filtraje multimomento y puede ser revisado con detalle en $[18,19]$.

En el régimen $2 \mathrm{MM}$ durante cada ciclo de filtraje se procesan dos muestras provenientes de dos instantes de tiempo (procesamiento no instantáneo), por lo que el coeficiente de correlación entre las dos muestras adyacentes (denotado como $\rho$ ) es un parámetro de diseño. La ventaja de considerar régimen $2 \mathrm{MM}$, es que se pueden tener los beneficios del procesamiento multimomento prácticamente sin retardos significativos. 
En el régimen unimomento (1MM) durante cada ciclo se procesa una muestra de un solo instante de tiempo (procesamiento instantáneo), no aumenta la precisión del filtraje y es justamente el tipo de procesamiento característico del EKF y sus variantes listadas anteriormente.

\subsection{Modelo de simulación}

La implementación práctica de la metodología propuesta se llevó a cabo desarrollando un banco de pruebas de simulación basado en MatLab.

La metodología está compuesta por dos elementos:

1) Una estructura de filtraje dinámico no lineal basada en el filtro de Kalman (EKF o SKF según sea el caso).

2) Modelaje apropiado de la señal débil real congruente con la estructura de filtraje a usar (EKF o SKF).

Para la simulación mediante MatLab se usa la versión discreta del filtro de Kalman y para dar continuidad a la exposición se reproduce a continuación. La dinámica de estados en un sistema discreto está dada por:

$$
\begin{gathered}
x_{k+1}=f\left(x_{k}\right)+\varepsilon_{k} \\
y_{k}=s\left(x_{k}\right)+n_{0 k s}
\end{gathered}
$$

donde $x_{k}$ representa el estado del sistema y $y_{k}$ una medición del estado del sistema, $\left\{n_{0 k}\right\}$ y $\left\{\varepsilon_{k}\right\}$ son procesos independientes de ruido blanco Gaussiano con media cero y matrices de covarianza

$$
E\left[n_{0 k} n_{0 k}^{T}\right]=N_{0 k} \quad y \quad E\left[\varepsilon_{k} \varepsilon_{k}^{T}\right]=Q_{k}
$$

respectivamente, $Q_{k}$ denota ruido de proceso y ruido de medición. Los ciclos de predicción y corrección del filtro de Kalman están dados por:

$$
\begin{gathered}
\text { Predicción }\left\{\begin{array}{c}
\hat{x}_{k+1}^{-}=f\left(x_{k}^{+}\right) \\
P_{k}^{-}=A_{k} P_{k}^{+} A_{k}^{T}+Q_{k}
\end{array}\right. \\
\text { Corrección }\left\{\begin{array}{c}
G_{k}=P_{k}^{-} H_{k}^{T}\left[H_{k} P_{k}^{-} H_{k}^{T}+N_{0 k}\right]^{-1} \\
\hat{x}_{k}^{+}=\hat{x}_{k}^{-}+G k\left[y_{k}-s\left(x_{k}^{-}\right)\right] \\
P_{k}^{+}=P_{k}^{-}+G_{k} H_{k} P_{k}^{-}
\end{array}\right.
\end{gathered}
$$

donde $G_{k}$ es la ganancia de Kalman, $\hat{x}_{k}^{-}$es el estimado a priori del estado en el $k$-ésimo ciclo de actualización, $\hat{x}_{k}^{+}$es el estimado a posteriori del estado en el $k$-ésimo ciclo de actualización, $P_{k}^{-}, P_{k}^{+}$son respectivamente las estimaciones a priori y a posteriori de las matrices de covarianza en la $k$-ésima etapa, $A_{k}$ es la matriz de linealización (o de transición de estados) y $H_{k}$ es la matriz que indica la relación entre la medición y el vector de estado en el ciclo $k$-ésimo suponiendo ausencia de ruido. Para el caso del SKF $A_{k}$ es una matriz fija en cada ciclo mientras que para el EKF la matriz se actualiza en cada ciclo mediante el cálculo jacobiano:

$$
A_{k}=\frac{\partial f\left(x_{k}\right)}{\partial x_{k}}
$$

$\mathrm{Al}$ usar el EKF la estructura del filtro está dada por (7), para la función $f\left(x_{k}\right)$ se usa (2), (3) ó (4) y la linealización según (8). Aquí se modela la señal débil real usando alguno de los procesos caóticos (2)-(4), es decir, se analiza cuál de las componentes $(x, y$ o $z)$ de (2), (3) o (4) es la más adecuada para ser usada como modelo. Para este propósito, primero el tiempo de muestreo $(T s)$ de las ecuaciones caóticas discretas se modifica hasta lograr una coincidencia entre las variaciones temporales de la componente del atractor caótico seleccionado y la señal real (hacer que las escalas de tiempo sean lo más cercanas posible). Segundo la señal deseada se normaliza en relación con la media y la varianza de la componente del atractor. En $[18,19]$ se ha demostrado que la componente $\mathrm{x}$ de los tres atractores caóticos (2)-(4) es la más adecuada para modelar señales experimentales. Después de llevar a cabo el mismo análisis de modelaje se encontró que el mismo criterio es aplicable para las señales fenomenológicas de este trabajo.

Cuando se usa el SKF la matriz $A_{k}$ es fija y el modelaje de la señal real debe reflejarse justamente en $A_{k}$. Con este propósito se emplea la herramienta de identificación de sistemas de MatLab que denotaremos como SIT [20] (System Identification Toolbox). Dicha herramienta está basada en la teoría de identificación de sistemas [21]. Para identificar a la señal real (sísmica, ECG, tipo voz, RFI) la SIT de MatLab analiza sus propiedades espectrales y arroja como modelo una matriz constante.

La parte experimental de la siguiente sección muestra que es posible usar dicha matriz justamente como una matriz de linealización fija en la estructura de filtraje dada por (7), lo cual es en realidad solo una aproximación del procedimiento de linealización instantáneo que se requiere para el filtraje cuasióptimo cuando se usa la LGA.

Para hacer una comparación justa con el EKF, de dimensión 3 según las fórmulas (2)-(4), se diseña un SKF tridimensional. Para obtener la matriz $A_{k}$ mediante la SIT de MatLab se usa el comando «ident». La identificación (de la señal sin ruido) se hace seleccionando la opción «state space models» [7,22] para el caso tridimensional. El programa ofrece tres opciones de estimación (método de subespacio, reducción regularizada y minimización del error de predicción) y al final indica el porcentaje de confianza para cada opción. Experimentalmente se encontró que la opción de minimización del error de predicción ofrece el mejor 
porcentaje de confianza para la estimación de la matriz.

En la metodología propuesta aquí la identificación de sistemas es justamente un modelaje de la señal real. Se hace la identificación para un vector (lo más grande posible) de la señal real (sin ruido). Suponiendo que las señales bajo estudio son estacionarias, la matriz de identificación de sistema puede considerarse como la matriz de linealización fija $A_{k} \mathrm{y}$, por lo tanto, ser un modelo de señal para cualquier otro vector más corto de la misma señal fenomenológica. Al usar la matriz de identificación en la estructura del SKF se logra un procesamiento con información a priori y con datos experimentales.

Para lograr una mayor fidelidad de filtraje se emplea el régimen 2MM. Tal y como se comenta en [19] las soluciones cuasi óptimas (en este caso para algoritmos multimomento) tienen como base alguna heurística que puede incorporar conocimiento y/o estructuras previas. Siendo así, el régimen $2 \mathrm{MM}$ que se ha utilizado en este trabajo tiene la forma:

$$
\begin{gathered}
\hat{x}_{k+1}^{-}=f\left(\hat{x}_{k}^{+}\right) \\
P_{k}^{-}=A_{k} P_{k}^{+} A_{k}^{T}+Q_{k} \\
G_{k}=P_{k}^{-} H_{k}^{T}\left[H_{k} P_{k}^{-} H_{k}^{T}+N_{0 k}\right]^{-1} \\
\hat{x}_{k}^{+}=\hat{x}_{k}^{-}+G_{k}\left[y_{k}-s\left(x_{k}^{-}\right)\right] \\
P_{k}^{+}=P_{k}^{-}+G_{k} H_{k} P_{k}^{-}
\end{gathered}
$$

Donde el subíndice 2 del lado izquierdo de cada variable denota una variable $2 \mathrm{MM}$. Para este algoritmo de filtraje se tiene en la columna izquierda la estructura dada por (7) y en la columna derecha la única diferencia es que la última operación del ciclo de corrección no existe, ambas columnas operan en paralelo. En (7) la salida del filtro es $\hat{x}_{k}^{+}$mientras que en (9) es ${ }_{2} \hat{x}_{k+1}^{-}$. En el régimen $2 \mathrm{MM}$ en la columna del lado derecho en la última operación del ciclo de predicción se calcula el estimado a priori de la matriz de covarianza tomando en cuenta el coeficiente de correlación $\rho$ de las dos muestras. Obsérvese que la estructura (9) puede operar tanto para el EKF como para el SKF siguiendo las observaciones descritas en párrafos anteriores.

En la siguiente sección se muestra de manera experimental la eficiencia del filtraje aquí propuesto considerando señales de naturaleza significativamente diferente, tales como, señales sísmicas, señales electrocardiográficas fetales (FECG), señales tipo voz y señales de interferencia de radiofrecuencia (RFI). Tales señales pueden considerarse caóticas $[1,2,12,15,23,24]$

\section{Resultados y discusión}

Los siguientes resultados muestran el filtraje mediante SKF y EKF tanto para el régimen 1MM como para 2MM. En el régimen 2MM el parámetro $\rho$ determina una diferente fidelidad en el filtraje. Si $\rho=0$, se tiene el régimen $1 \mathrm{MM}$ ( $\sin$ incremento en la fidelidad). $\mathrm{Si}$ $\rho=1$, vea fórmula (9), se tiene una condición de singularidad con una matriz de covarianza igual a cero (fidelidad que tiende $a+\infty$ ).

Se escogió $\rho=0.85$ para un análisis homogéneo de resultados.
Las figuras muestran las curvas traslapadas de la señal original (sin ruido) y la señal después filtrarse con el esquema de filtraje y régimen correspondiente que se indica en cada figura. Las figuras muestran solo un caso de umbral de señal débil cuando la $S N R=-3 d B$. Manipulando $N_{0}$ para analizar diferentes umbrales de señales débiles $(S N R \leq 0 d B)$, las tablas 2-5 muestran el desempeño del SKF y EKF bajo los regímenes 1MM y $2 \mathrm{MM}$ en términos del NMSE (descrito en párrafos de la introducción). También se muestran los tiempos promedio (en segundos) que se requieren para procesar 5000 muestras para cada uno de los fenómenos estudiados y sus correspondientes filtrajes.

Tanto para el SKF como el EKF se hace un modelaje imperfecto de la señal fenomenológica (existe un cierto grado de incertidumbre en las condiciones iniciales para el filtraje), por eso un valor de ruido de proceso débil, vea $Q$ en (7) y (9), debe incluirse en la estructura de filtraje (valor de $Q$ indicado en las tablas 2-5).

Para las señales sísmicas no se presentan resultados del SKF porque no fue posible obtener la correspondiente matriz de linealización fija (matriz de identificación de sistemas) ya que dichas señales tienen una duración muy limitada para un adecuado análisis espectral mediante la SIT.

\subsection{Experimento 1. Señales electrocardiográfi- cas fetales (FECG)}

Los datos experimentales se obtuvieron de la base de datos de PhysioNet [25]. La señal para este experimento corresponde al corazón de un producto fetal en la semana 36 de gestación. Para una $S N R=-3 d B$ la Figura 1 muestra la señal original y la señal filtrada usando el EKF 1MM con la componente $x$ de Rossler como modelo. La Tabla 2 muestra los resultados completos. 


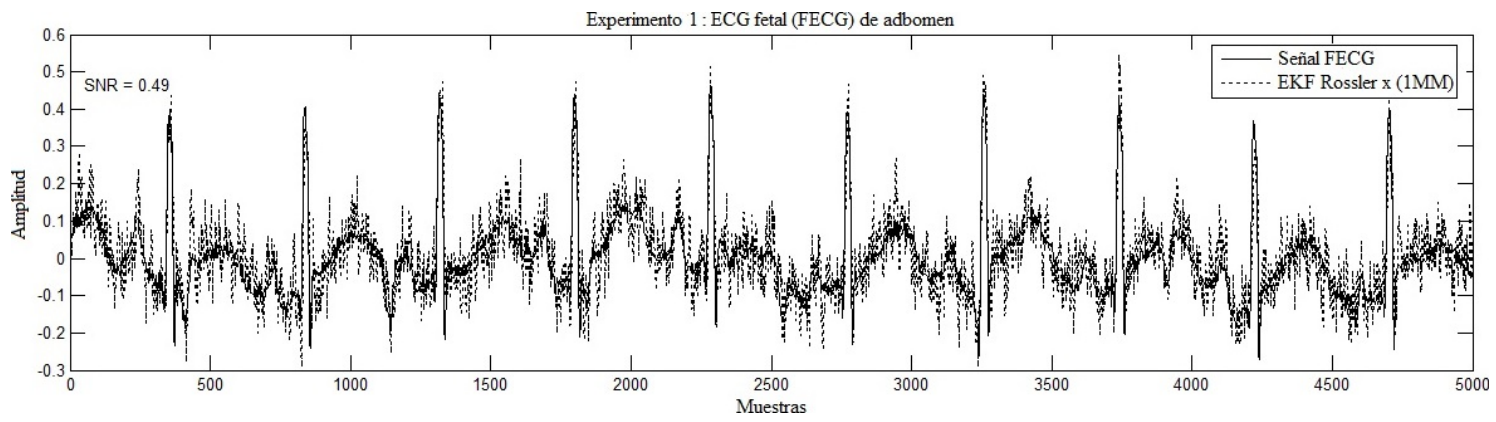

Figura 1. Señales en el experimento 1

Tabla 2. Resultados del NMSE para el experimento 1

\begin{tabular}{ccccc}
\hline SNR & $0 \mathrm{~dB}$ & $-3 \mathrm{~dB}$ & $-10 \mathrm{~dB}$ & Tiempo de procesamiento \\
\hline \multicolumn{5}{c}{ SKF $Q=0.04\left(\right.$ con matriz de linealización $\left.A_{k}\right)$} \\
\hline $1 \mathrm{MM}$ & 0.0025 & 0.0037 & 0.0078 & $0.43 \mathrm{~s}$ \\
$2 \mathrm{MM}$ & 0.0021 & 0.0032 & 0.0065 & $0.89 \mathrm{~s}$ \\
\hline \multicolumn{5}{c}{ EKF Rossler x $Q=0.21$} \\
\hline $1 \mathrm{MM}$ & 0.0026 & 0.0040 & 0.0098 & $1.825 \mathrm{~s}$ \\
$2 \mathrm{MM}$ & 0.0023 & 0.0036 & 0.0079 & $3.503 \mathrm{~s}$ \\
\hline \multicolumn{5}{c}{ EKF Lorenz x $Q=0.42$} \\
\hline $1 \mathrm{MM}$ & 0.0029 & 0.0042 & 0.010 & $1.782 \mathrm{~s}$ \\
$2 \mathrm{MM}$ & 0.0023 & 0.0034 & 0.0083 & $3.59 \mathrm{~s}$ \\
\hline \multicolumn{5}{c}{ EKF Chua x $Q=0.075$} \\
\hline $1 \mathrm{MM}$ & 0.0034 & 0.0053 & 0.015 & $1.812 \mathrm{~s}$ \\
$2 \mathrm{MM}$ & 0.0026 & 0.0042 & 0.012 & $3.61 \mathrm{~s}$ \\
\hline
\end{tabular}

\subsection{Experimento 2. Señales de tipo voz}

Para este experimento se usaron sonidos sostenidos de vocales (vocal «o»). Este tipo de señales se usan para procedimientos de síntesis de voz [23]. La Figura 2 muestra con línea continua el sonido sostenido de la vocal «o» (grabado durante 5 segundos a
$22050 \mathrm{~Hz}$ ) y con línea punteada la señal filtrada usando el SKF 2MM con su matriz evaluada mediante el SIT. Los resultados (muy semejantes al experimento anterior) se muestran en la Tabla 3. Para este experimento ninguna de las componentes del atractor de Lorenz resultó adecuada para modelar las señales tipo voz.

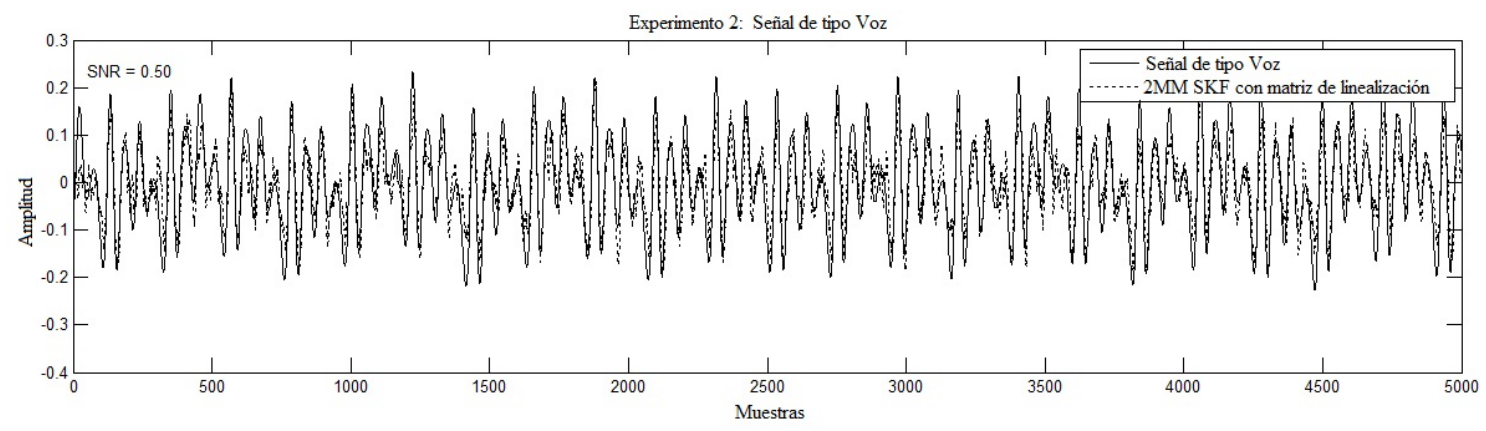

Figura 2. Señales en el experimento 2. 
Tabla 3. Resultados del NMSE para el experimento 2

\begin{tabular}{ccccc}
\hline SNR & $0 \mathrm{~dB}$ & $-3 \mathrm{~dB}$ & $-10 \mathrm{~dB}$ & Tiempo de procesamiento \\
\hline \multicolumn{5}{c}{ SKF $\mathrm{Q}=0.0081\left(\right.$ con matriz de linealización $\left.A_{k}\right)$} \\
\hline $1 \mathrm{MM}$ & 0.0025 & 0.0037 & 0.0079 & $0.47 \mathrm{~s}$ \\
$2 \mathrm{MM}$ & 0.0015 & 0.0024 & 0.0053 & $0.95 \mathrm{~s}$ \\
\hline \multicolumn{5}{c}{ EKF Rossler x $\mathrm{Q}=0.23$} \\
\hline $1 \mathrm{MM}$ & 0.0029 & 0.0044 & 0.0124 & $1.792 \mathrm{~s}$ \\
$2 \mathrm{MM}$ & 0.0027 & 0.0039 & 0.011 & $3.611 \mathrm{~s}$ \\
\hline \multicolumn{5}{c}{ EKF Chua x $\mathrm{Q}=0.76$} \\
\hline $1 \mathrm{MM}$ & 0.0031 & 0.0048 & 0.0137 & $1.81 \mathrm{~s}$ \\
$2 \mathrm{MM}$ & 0.0025 & 0.0043 & 0.0130 & $3.58 \mathrm{~s}$ \\
\hline
\end{tabular}

\subsection{Experimento 3. Señales sísmicas}

Para este experimento se usó un simulador de MatLAb basado en los modelos sísmicos reportados en [26]. Para una $S N R=-3 d B$, la Figura 3 muestra una señal sís- mica y su versión filtrada usando el EKF 2MM con la componente x de Rossler como modelo. Los resultados completos se muestran en la Tabla 4. Para la señal sísmica no fue posible obtener la matriz de linealización por lo que no se reporta el SKF para este caso.



Figura 3. Señales en el experimento 3.

Tabla 4. Resultados del NMSE para el experimento 3

\begin{tabular}{lcccc}
\hline SNR & $0 \mathrm{~dB}$ & $-3 \mathrm{~dB}$ & $-10 \mathrm{~dB}$ & Tiempo de procesamiento \\
\hline \multicolumn{5}{c}{ EKF Rossler x Q $=0.35$} \\
\hline $1 \mathrm{MM}$ & 0.0048 & 0.0074 & 0.0178 & $1.79 \mathrm{~s}$ \\
$2 \mathrm{MM}$ & 0.0047 & 0.0073 & 0.0135 & $3.53 \mathrm{~s}$ \\
\hline \multicolumn{5}{c}{ EKF Lorenz x Q $=0.135$} \\
\hline $1 \mathrm{MM}$ & 0.0058 & 0.0093 & 0.0245 & $1.807 \mathrm{~s}$ \\
$2 \mathrm{MM}$ & 0.0054 & 0.0081 & 0.0187 & $3.62 \mathrm{~s}$ \\
\hline \multicolumn{5}{c}{ EKF Chua x Q 0.135} \\
\hline $1 \mathrm{MM}$ & 0.0057 & 0.0095 & 0.029 & $1.816 \mathrm{~s}$ \\
$2 \mathrm{MM}$ & 0.0051 & 0.0084 & 0.023 & $3.65 \mathrm{~s}$ \\
\hline
\end{tabular}




\subsection{Experimento 4: Señales de RFI}

Este experimento considera la RFI generada por equipo de cómputo $[24,27]$ y que afecta la transmisión de señales de información deseadas. Para una
$S N R=-3 d B$ la Figura 4 muestra la señal de RFI y su versión filtrada usando el SKF $1 \mathrm{MM}$ con su matriz de linealización evaluada mediante el SIT. La Tabla 5 muestra los resultados completos.



Figura 4. Señales en el experimento 4.

Tabla 5. Resultados del NMSE para el experimento 4

\begin{tabular}{ccccc}
\hline SNR & $0 \mathrm{~dB}$ & $-3 \mathrm{~dB}$ & $-10 \mathrm{~dB}$ & Tiempo de procesamiento \\
\hline \multicolumn{5}{c}{ SKF Q $=0.02\left(\right.$ con matriz de linealización $\left.A_{k}\right)$} \\
\hline $1 \mathrm{MM}$ & 0.0018 & 0.003 & 0.0098 & $0.51 \mathrm{~s}$ \\
$2 \mathrm{MM}$ & 0.0015 & 0.0025 & 0.0085 & $0.92 \mathrm{~s}$ \\
\hline \multicolumn{5}{c}{ EKF Rossler x Q $=0.2$} \\
\hline $1 \mathrm{MM}$ & 0.0026 & 0.005 & 0.019 & $1.872 \mathrm{~s}$ \\
$2 \mathrm{MM}$ & 0.0023 & 0.0036 & 0.011 & $3.9 \mathrm{~s}$ \\
\hline \multicolumn{5}{c}{ EKF Lorenz x Q $=0.6$} \\
\hline $1 \mathrm{MM}$ & 0.0023 & 0.0032 & 0.04 & $1.76 \mathrm{~s}$ \\
$2 \mathrm{MM}$ & 0.0016 & 0.0027 & 0.0083 & $3.81 \mathrm{~s}$ \\
\hline \multicolumn{5}{c}{ EKF Chua x Q $=0.4$} \\
\hline $1 \mathrm{MM}$ & 0.0034 & 0.0053 & 0.035 & $1.86 \mathrm{~s}$ \\
$2 \mathrm{MM}$ & 0.0026 & 0.0042 & 0.029 & $3.77 \mathrm{~s}$ \\
\hline
\end{tabular}

De las tablas se observa que el método $2 \mathrm{MM}$ muestra un NMSE ligeramente mejor. Todas las metodologías de filtraje presentadas son bastante efectivas, ya que se caracterizan por un valor muy bajo del NMSE. En el escenario de $S N R=-10 d B$ (una señal extremadamente débil) es virtualmente imposible distinguir visualmente (no se muestra en figuras por razones de espacio) las señales deseadas de entre el ruido, sin embargo, el valor del NMSE tiene valores de alrededor del $1 \%$ para el filtraje mediante SKF y para el EKF, tanto para la metodología 1MM como para 2MM.

Hay que tener en cuenta que la metodología 2MM consume más tiempo con respecto a la metodología $1 \mathrm{MM}$, sin embargo, no es más del doble de tiempo. El filtraje mediante SKF es (casi 3 veces) más rápido porque no hay proceso de linealización. Los tiempos de procesamiento junto con la complejidad y fidelidad del filtraje deben ser criterios de elección para escoger el algoritmo de filtraje adecuado para cada implementación concreta.

El SKF con matriz de linealización fija (modelando la señal de interés mediante una matriz de identificación de sistema) muestra los mejores resultados, lo cual indica que para el procesamiento de algoritmos cuasilineales de filtraje, la influencia de las propiedades espectrales de los datos de entrada prevalece sobre la influencia de las estadísticas no gaussianas. Los valores del NMSE obtenidos en las simulaciones son muy semejantes para el filtraje de las diferentes señales, por lo que en la práctica no es realmente determinante el modelo particular del atractor caótico o la matriz 
de linealización (obtenida a partir de la SIT) que se aplica.

¿Por qué ocurre esto? Todos los atractores caóticos que hemos empleado para modelar las señales reales, generan caos como un proceso estocástico cuasideterminista. Por eso todos los algoritmos de filtraje cuasi óptimo mencionados antes (incluido el EKF y sus modificaciones) que aplican la idea del modelaje caótico, operan en un régimen muy próximo a la singularidad, es decir, la forma de la PDF a posteriori se concentra alrededor de la PDF a priori de la señal deseada independientemente del valor de la SNR $[18,19]$ y eso justamente permite obtener valores tan bajos del NMSE para señales muy débiles (SNR menores a 0 $\mathrm{dB}$ y hasta $-10 \mathrm{~dB}$ ). Así que, para el filtraje de alta fidelidad, el término lineal de la expansión en series de Taylor para el algoritmo cuasilineal $[8,9,22]$ es significativamente más determinante que los términos relacionados a las no linealidades (matriz jacobiana, etc.), es decir, la aproximación lineal es suficiente.

\section{Conclusiones}

Para el procesamiento de señales débiles se ha propuesto utilizar una estructura de filtraje simple y robusta que está basada en el filtro de Kalman. Esta estructura permite la incorporación del régimen $2 \mathrm{MM}$ lo cual mejora la precisión del filtraje.

Para señales fenomenológicas no gaussianas, dependiendo del escenario específico, de los requerimientos de complejidad computacional y error tolerable, se puede recurrir al EKF usando señales caóticas como modelo de las señales reales. O bien, se puede recurrir al SKF con una matriz de linealización fija, es decir, se usa la herramienta SIT de MatLab para evaluar la matriz de identificación de sistemas y se usa como modelo de la señal real. Esto permite un «grado significativo de libertad» para el diseño del bloque de filtraje.

Los resultados experimentales muestran gran precisión en el filtraje de señales débiles para todos los escenarios aquí considerados y dada la naturaleza tan diversa de dichos escenarios muy probablemente se pueda aplicar en otros escenarios (trabajo futuro).

\section{Referencias}

[1] Y. Li, B. Yang, Y. Yuan, X. Zhao, and H. Lin, "Ability to detect weak effective seismic signals by utilizing chaotic vibrator system," Chinese Science Bulletin, vol. 51, pp. 3010-3017, 2006. [Online]. Available: https://doi.org/10.1007/s11434-006-2191-0

[2] Y. Li, B. J. Yang, J. Badal, X. P. Zhao, H. B. Lin, and R. L. Li, "Chaotic system detection of weak seismic signals," Geophysical Journal International, vol. 178, no. 3, pp. 1493-1522, 2009. [Online]. Available: https: //doi.org/10.1111/j.1365-246X.2009.04232.x

[3] J. Shu-Yao, Y. Fei, C. Ke-Yu, and C. En, "Application of stochastic resonance technology in underwater acoustic weak signal detection," in OCEANS 2016 - Shanghai, April 2016, pp. 1-5. [Online]. Available: https: //doi.org/10.1109/OCEANSAP.2016.7485567

[4] S. L. Joshi, R. A. Vatti, and R. V. Tornekar, "A survey on ECG signal denoising techniques," in 2013 International Conference on Communication Systems and Network Technologies, April 2013, pp. 60-64. [Online]. Available: https://doi.org/10.1109/CSNT.2013.22

[5] H. Li, R. Wang, S. Cao, Y. Chen, N. Tian, and X. Chen, "Weak signal detection using multiscale morphology in microseismic monitoring," Journal of Applied Geophysics, vol. 133, pp. 39-49, 2016. [Online]. Available: https://doi.org/10.1016/j.jappgeo.2016.07.015

[6] R. Han, J. Li, G. Cui, X. Wang, W. Wang, and X. Li, "Seismic signal detection algorithm based on GS transform filtering and EMD denoising," in 2018 IEEE 4th International Conference on Computer and Communications (ICCC), Dec 2018, pp. 1213-1217. [Online]. Available: https: //doi.org/10.1109/CompComm.2018.8781035

[7] H. Van Trees, Detection, Estimation, and Modulation Theory: Detection, Estimation, and Linear Modulation Theory, 2001. [Online]. Available: https://doi.org/10.1002/0471221082

[8] A. Jazwinski, Stochastic Processes and Filtering Theory. Academic Press, 1970. [Online]. Available: https://bit.ly/34iDlnz

[9] R. L. Stratonovich, Topics of the Theory of Random Noise, 1967. [Online]. Available: https://bit.ly/2PcK2DB

[10] W.-R. Wu and A. Kundu, "Image estimation using fast modified reduced update kalman filter," IEEE Transactions on Signal Processing, vol. 40, no. 4, pp. 915-926, April 1992. [Online]. Available: https://doi.org/10.1109/78.127963

[11] R. Parseh, K. Kansanen, and D. Slock, "Distortion outage analysis for joint space-time coding and kalman filtering," IEEE Transactions on Signal Processing, vol. 65, no. 9, pp. 2291-2305, May 2017. [Online]. Available: https://doi.org/10.1109/TSP.2017.2655493

[12] M. de Sousa Vieira, "Chaos and synchronized chaos in an earthquake model," 
Physical Review Letters, vol. 82, pp. 201204, Jan 1999. [Online]. Available: http: //doi.org/10.1103/PhysRevLett.82.201

[13] G. Kolumban, M. P. Kennedy, and L. O. Chua, "The role of synchronization in digital communications using chaos. I. fundamentals of digital communications," IEEE Transactions on Circuits and Systems I: Fundamental Theory and Applications, vol. 44, no. 10, pp. 927-936, Oct 1997. [Online]. Available: https://doi.org/10.1109/81.633882

[14] G. Kaddoum, "Design and performance analysis of a multiuser ofdm based differential chaos shift keying communication system," IEEE Transactions on Communications, vol. 64, no. 1, pp. 249-260, Jan 2016. [Online]. Available: https://doi.org/10.1109/TCOMM.2015.2502259

[15] N. V. Thakor, "Chaos in the heart: signals and models," in Proceedings of the 1998 2nd International Conference Biomedical Engineering Days, May 1998, pp. 11-18. [Online]. Available: https://doi.org/10.1109/IBED.1998.710541

[16] P. J. García-Laencina and G. RodríguezBermúdez, "Analysis of EEG signals using nonlinear dynamics and chaos: A review," Applied Mathematics $\&$ Information Sciences, pp. 2309-2321, 2015. [Online]. Available: https://bit.ly/2Pg2NGa

[17] V. S. Anishchenko, V. Astakhov, A. Neiman, T. Vadivasova, and L. Schimansky-Geier, Nonlinear Dynamics of Chaotic and Stochastic Systems. Springer-Verlag Berlin Heidelberg, 2007. [Online]. Available: http: //doi.org/10.1007/978-3-540-38168-6

[18] V. Kontorovich and Z. Lovtchikova, "Nonlinear filtering of chaos for real time applications," in Selected Topics in Nonlinear Dynamics and Theoretical Electrical Engineering, K. Kyamakya, W. A. Halang, W. Mathis, J. C. Chedjou, and Z. Li, Eds. Berlin, Heidelberg: Springer Berlin Heidelberg, 2013, pp. 41-59. [Online]. Available: https://doi.org/10.1007/978-3-642-37781-5_3

[19] V. Kontorovich, Z. Lovtchikova, and F. RamosAlarcon, Nonlinear Filtering of Weak
Chaotic Signals, 2017. [Online]. Available: http://doi.org/10.5772/intechopen.70717

[20] E. Nikulchev, "Simulation of robust chaotic signal with given properties," Advanced Studies in Theoretical Physics, vol. 8, no. 21, pp. 939-944, 2014. [Online]. Available: http://dx.doi.org/10.12988/astp.2014.48106

[21] L. Ljung, System Identification: Theory for the user. Prentice Hall PTR, 1999. [Online]. Available: https://bit.ly/2YIDEaj

[22] V. Pugachev and I. Sinitsyn, Stochastic Differential systems Analysis and Filtering. John Wiley \& Sons, 1987. [Online]. Available: https://bit.ly/2qKkrZc

[23] M. Banbrook, S. McLaughlin, and I. Mann, "Speech characterization and synthesis by nonlinear methods," IEEE Transactions on Speech and Audio Processing, vol. 7, no. 1, pp. 1-17, Jan 1999. [Online]. Available: https://doi.org/10.1109/89.736326

[24] D. Ilitzky Arditti, A. Alcocer Ochoa, V. Kontorovich Mazover, and F. Ramos Alarcon Barroso, "Adaptive mitigation of platformgenerated radio-frequency interference," Patent US 2015.0 051 880A1, 2015. [Online]. Available: https://bit.ly/2Pesh6G

[25] Physionet. (2019) Physiobank atm. [Online]. Available: https://bit.ly/2LMxcKf

[26] F. R. Rofooei, A. Mobarake, and G. Ahmadi, "Generation of artificial earthquake records with a nonstationary kanai-tajimi model," Engineering Structures, vol. 23, no. 7, pp. 827-837, 2001. [Online]. Available: https://doi.org/10.1016/S0141-0296(00)00093-6

[27] E. X. Alban, M. E. Magana, H. G. Skinner, and K. P. Slattery, "Statistical modeling of the interference noise generated by computing platforms," IEEE Transactions on Electromagnetic Compatibility, vol. 54, no. 3, pp. 574-584, June 2012. [Online]. Available: https://doi.org/10.1109/TEMC.2011.2164255 